\title{
Maximum likelihood approach to DoA estimation using lens antenna array
}

\author{
Zheng-Ming Jiang $^{1}$, Peichang Zhang ${ }^{1 *}$, Mohamed Rihan ${ }^{1,2}$, Lei Huang ${ }^{1}$ and Jihong Zhang ${ }^{1}$
}

\begin{abstract}
Massive antenna array has been proposed to improve the spectral efficiency and link reliability in wireless communication systems. However, using large antenna arrays incurs additional cost in terms of signal processing and hardware complexity. The electromagnetic (EM) lens-focusing antennas are introduced as a promising technique to reduce the hardware complexity and cost. On the other hand, determining the location of users in terms of their direction-of-arrival (DoA) using these lens array becomes of great interest for different $5 \mathrm{G}$ services. This paper addresses the issue of DoA estimation by adopting lens antenna array (LNA). We firstly derive an expression for the received signal with the adoption of LNA, and then a maximum likelihood (ML) estimator for the DoA has been obtained. Depending on the ability of the lens array to focus the signal power on a subset of antennas as a function of DoA. We propose using the antenna selection (AS) technology to select an antenna subset aiming to reduce the number of radio frequency (RF) chains and accordingly reducing the hardware cost. The simulation results show the the capability of the proposed method to avoid the phase ambiguity problem and provide high accurate DoA estimation of signals.
\end{abstract}

Keywords: Maximum likelihood estimation, Lens antenna, Antenna selection, Phase ambiguity

\section{Introduction}

With the advent of 5G communications, the demands for low latency and high data rates significantly increase [1-3]. Among the technologies that have been proposed to achieve these goals is the millimeter wave ( $\mathrm{mm}$-Wave)based massive antenna array system [4]. It has been shown that massive antenna arrays can improve both link reliability and spectral efficiency of the system. However, as the number of antennas increases, a number of challenging problems comes out and it needs to be considered. Firstly, employing massive number of antennas needs a large number of RF chains, which may lead to both higher energy consumption of system and higher hardware cost and complexity. This is because the RF chains composed of digital to analog (D/A) converters, analog to digital (A/D) converters, amplifiers, and mixers. On the other hand, directly processing the data received from massive number of antennas causes heavy computational load, which may impose a big challenge in practical application.

*Correspondence: pzhang@szu.edu.cn

${ }^{1}$ Guangdong Laboratory of Artificial-Intelligence and Cyber- Economics (SZ),

Shenzhen University, Shenzhen 518060, People's Republic of China

Full list of author information is available at the end of the article
In order to solve these issues, various methodologies have been suggested. In [5], the authors have proposed using multiple antennas combining architecture to reduce the power consumption. In [6] and [7], the electromagnetic lens-focusing antennas are used to reduce the hardware complexity and accordingly minimize the computational cost. An antenna selection algorithm was proposed in [8] to decrease the number of RF chains. Antenna selection techniques can effectively reduce both the hardware cost as well as the number of RF chains. However, the problem of DoA estimation for lens antenna array (LNA)based systems has not yet been addressed in the literature. Actually, it is widely believed that estimating the DoAs is a key aspect for future communication systems. In particular, the $5 G$ public-private-partnership automotive vertical white paper has proposed the requirement of highly accurate location [2] for different 5G services. Location information is one of the eight key performance indicators ${ }^{1}$ required for different vertical industries like autonomous vehicles, e-health, and media sectors. Therefore, to meet

\footnotetext{
${ }^{1}$ The eight key performance indicators are end-to-end latency, reliability, data rate, communication range, node mobility, network density, position accuracy, and security.
}

\section{Springer Open}

(c) The Author(s). 2019 Open Access This article is distributed under the terms of the Creative Commons Attribution 4.0 International License (http://creativecommons.org/licenses/by/4.0/), which permits unrestricted use, distribution, and reproduction in any medium, provided you give appropriate credit to the original author(s) and the source, provide a link to the Creative Commons license, and indicate if changes were made. 
the convergence requirement for $5 \mathrm{G}$ industries, accurate estimation of DoA becomes a necessity.

There have been a number of methods for DoA estimation, such as multiple signal classification (MUSIC) [9], maximum likelihood (ML) estimation [10], and so on. However, all these methods require that the spacing of array elements must be not larger than half-wavelength, or it would cause the angle ambiguity problem. Against this background, this paper is the first that proposes the use of LNA for solving the angle ambiguity problem, where we have verified that the receive array response vector of LNA is approximately a sinc function of the DoA, which is an aperiodic function. Although the array structure with LNA can solve the phase ambiguity problem, large antenna arrays require increased signal processing complexity and hardware costs. In order to solve this problem, this paper makes full use of the distribution characteristics of the signal power and proposes maximal power criterion to select the corresponding antenna and two antennas around it. In order to estimate the angle of incidence of the signal, we just need to deal with the data on these three antennas. Specifically, we derive an expression for the received signal from massive LNA and accordingly formulate a ML approach to DoAs estimation for such LNA-based structure. Additionally, we study the use of LNA to avoid the angle ambiguity problem. Finally, we study the use of AS techniques with the LNA structure for reducing the number of RF chains and signal processing complexity.

The remainder of this paper is organized as follows. In Section 2, we derive an expression for the received signal from the LNA structure. In Section 3, we formulate the ML approach to the DoA estimation for the system that based on LNA. In Section 4, we compare the performance of ML method of the LNA structure, to that of the conventional schemes without the LNA. Conclusions are presented in Section 5.

\section{Problem formulation}

In optics, lens are able to change the direction of the light through refraction phenomena and accordingly focusing the energy of signal in that direction. A LNA is described in Fig. 1. We assume that the width of EM lens is $L$ and its thickness is negligible. The LNA has $N$ elements which

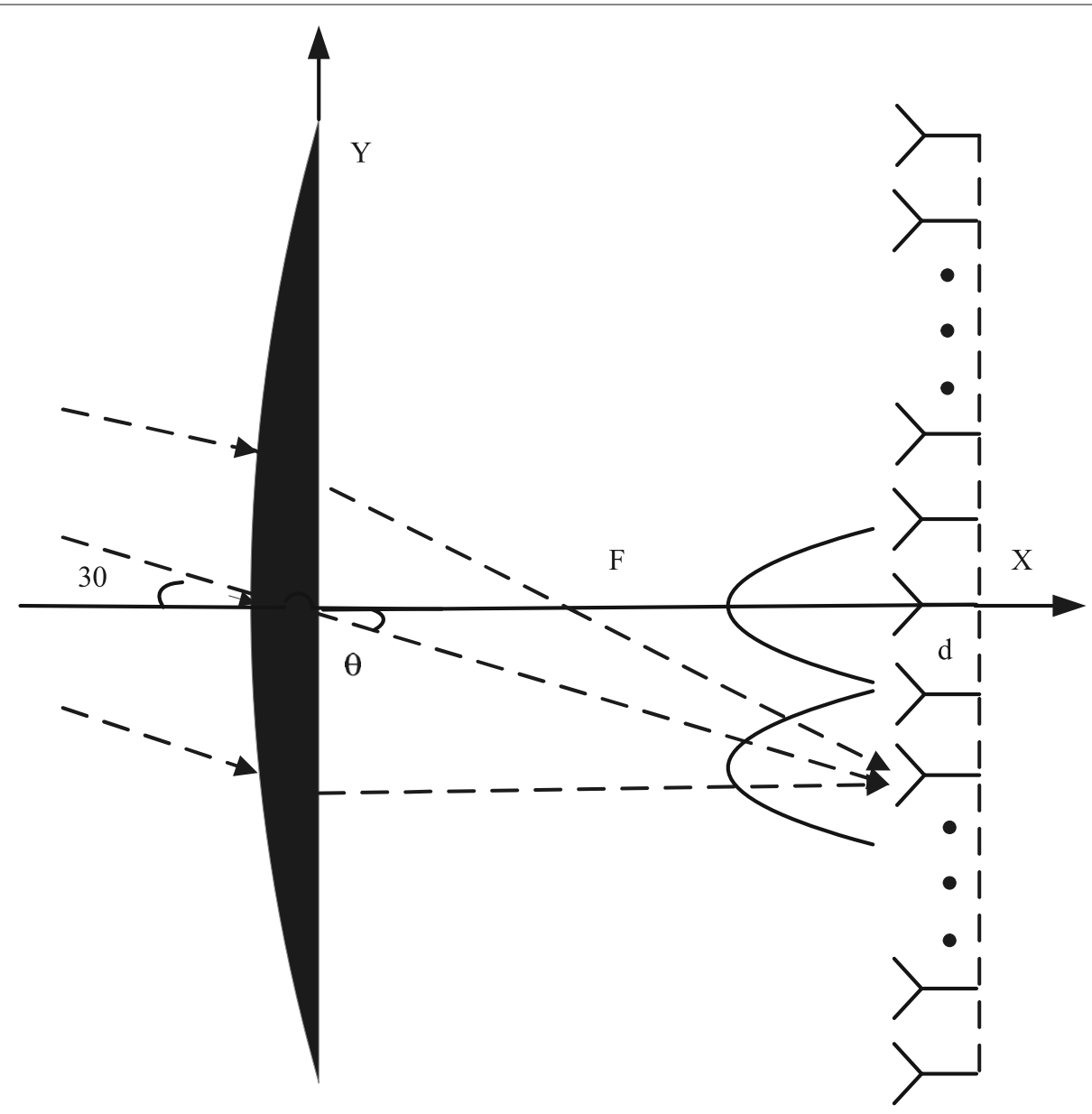

Fig. 1 Configuration of a LNA system 
are located on the $Y$-axis. The unit inter-element spacing $d$ is set to $\lambda / 2$, with $\lambda$ denoting the signal wavelength. The focal length of the lens is $F$, and the LNA locations relative to the lens center can be parameterized as $B_{m}\left(x_{m}=F, y_{m}=F \tan \left(\theta_{m}\right), z_{m}=0\right)$, where $\theta_{m} \in$ $[-\pi / 2, \pi / 2]$ is the angle of the $m$ th antenna element relative to the $x$-axis. The indices of each antenna element is set from $-(N-1) / 2$ to $(N-1) / 2$. Due to the characteristics of the EM lens, the light parallel to the axis will converge to the focus point $B_{0}$, with the same phase $\psi_{0}$. We assume that the phase of the signal reaching the lens is $\psi(y, z)$. With constructive superposition [11], we have

$$
\begin{array}{r}
\psi(y, z)+2 \pi / \lambda d\left(y, z, B_{0}\right)=\psi_{0} \\
\forall(y, z) \in\left[-D_{y} / 2, D_{y} / 2\right] \times\left[-D_{z} / 2, D_{z} / 2\right],
\end{array}
$$

where $d\left(y, z, B_{0}\right)=\sqrt{F^{2}+y^{2}+z^{2}}$ is the distance between the focus point $B_{0}$ and the point $(0, y, z)$ on the lens. With the phase shift designed such that, the phase delay from the lens input point $(0, y, z)$ to an arbitrary point $B_{m}\left(F, F \tan \left(\theta_{m}\right), 0\right)$ can be denoted as

$$
\psi(y, z)+\frac{2 \pi}{\lambda} d\left(y, z, B_{m}\right)=\psi_{m},
$$

by combining (1) and (2), we may obtain

$$
\begin{aligned}
\psi_{m} & =\psi_{0}+\frac{2 \pi}{\lambda} d\left(y, z, B_{m}\right)-\frac{2 \pi}{\lambda} d\left(y, z, B_{0}\right) \\
& =\psi_{0}+\frac{2 \pi}{\lambda} \sqrt{F^{2}+\left(F \tan \left(\theta_{m}\right)-y\right)^{2}+z^{2}}-\frac{2 \pi}{\lambda} \sqrt{F^{2}+y^{2}+z^{2}} .
\end{aligned}
$$

Setting $\psi_{m}=f(y)$, then we obtain

$$
\begin{aligned}
\frac{\partial f(y)}{\partial y} & =\frac{2 \pi}{\lambda} \frac{-F \sin \left(\theta_{m}\right) \cos \left(\theta_{m}\right)+y \cos \left(\theta_{m}\right)^{2}}{\cos \left(\theta_{m}\right) \sqrt{F^{2}-2 F y \sin \left(\theta_{m}\right) \cos \left(\theta_{m}\right)+y^{2} \cos \left(\theta_{m}\right)^{2}+z^{2} \cos \left(\theta_{m}\right)^{2}}} \\
& -\frac{2 \pi}{\lambda} \frac{y}{\sqrt{F^{2}+y^{2}+z^{2}}} .
\end{aligned}
$$

Using the first-order Taylor approximation, we get

$$
\begin{aligned}
f(y) & \approx f(0)+f^{\prime}(0)(y-0) \\
& =\psi_{0}+\frac{2 \pi}{\lambda} \sqrt{\frac{F^{2}}{\cos \left(\theta_{m}\right)^{2}}+z^{2}}-\frac{2 \pi}{\lambda} \sqrt{F^{2}+z^{2}} \\
& -\frac{2 \pi}{\lambda} \frac{F \sin \left(\theta_{m}\right)}{\sqrt{F^{2}+\cos \left(\theta_{m}\right)^{2} z^{2}}}(y-0) .
\end{aligned}
$$

By considering that $F \gg z$, we have $\sqrt{\frac{F^{2}}{\cos \left(\theta_{m}\right)^{2}}+z^{2}} \approx$ $\frac{F}{\cos \left(\theta_{m}\right)}$ and $\sqrt{F^{2}+z^{2}} \approx F, \frac{F \sin \left(\theta_{m}\right)}{\sqrt{F^{2}+\cos \left(\theta_{m}\right)^{2} z^{2}}}(y-0) \approx$ $y \sin \left(\theta_{m}\right)$. Inserting the obtained results into (5) yields

$$
f(y) \approx \psi_{0}+\frac{2 \pi}{\lambda}\left(\frac{F}{\cos \left(\theta_{m}\right)}-F-y \sin \left(\theta_{m}\right)\right) .
$$

We therefore assume that there is a uniform incident plane wave arriving at the lens with DoA $\phi$ as shown in Fig. 1.
By considering that the thickness of the lens is negligible, then we obtain

$$
h(y, z) \approx h(y),
$$

and

$$
h(y)=S e^{-j \frac{2 \pi}{\lambda} y \sin (\phi)} .
$$

According to the linear superposition principle [11], the signal received out of the the array can be formulated as

$$
Y\left(\theta_{m}\right)=\int_{-D_{z} / 2}^{D_{z} / 2} \int_{-D_{y} / 2}^{D_{y} / 2} h(y, z) e^{-j \psi_{m}} d_{y} d_{z} .
$$

Substituting (7) into (8), we obtain

$$
Y\left(\theta_{m}\right) \approx e^{-j \psi_{0}} D_{z} \int_{-D_{y} / 2}^{D_{y} / 2} h(y) e^{-j \frac{2 \pi}{\lambda}\left(\frac{F}{\cos \left(\theta_{m}\right)}-F-y \sin \left(\theta_{m}\right)\right)} d_{y} .
$$

Substituting (8) into (9), we obtain

$$
\begin{aligned}
Y\left(\theta_{m}\right) & =e^{-j \psi_{0}} D_{z} \int_{-D_{y} / 2}^{D_{y} / 2} S e^{-j \frac{2 \pi}{\lambda} y \sin (\phi)} e^{-j \frac{2 \pi}{\lambda}\left(\frac{F}{\cos \left(\theta_{m}\right)}-F-y \sin \left(\theta_{m}\right)\right)} d y \\
& =e^{-j \psi_{0}} e^{-j \frac{2 \pi}{\lambda} F \frac{1-\cos \left(\theta_{m}\right)}{\cos \left(\theta_{m}\right)}} D_{z} \int_{-D_{y} / 2}^{D_{y} / 2} S e^{-j \frac{2 \pi}{\lambda} y \sin (\phi)} e^{j \frac{2 \pi}{\lambda} y \sin \left(\theta_{m}\right)} d y \\
& =S e^{-j \psi_{0}} e^{-j \frac{2 \pi}{\lambda} F \frac{1-\cos \left(\theta_{m}\right)}{\cos \left(\theta_{m}\right)}} D_{z} \int_{-D_{y} / 2}^{D_{y} / 2} \cos (2 \pi / \lambda(\sin (\phi) \\
& \left.\left.-\sin \left(\theta_{m}\right)\right)\right) y-j \sin \left(2 \pi / \lambda\left(\sin (\phi)-\sin \left(\theta_{m}\right)\right)\right) y d_{y} .
\end{aligned}
$$

According to the symmetry of the integral, it follows from (10) that

$$
\begin{aligned}
Y\left(\theta_{m}\right) & =S e^{-j \psi_{0}} e^{-j \frac{2 \pi}{\lambda} F \frac{1-\cos \left(\theta_{m}\right)}{\cos \left(\theta_{m}\right)}} D_{z} \int_{-D_{y} / 2}^{D_{y} / 2} \cos \left(2 \pi / \lambda\left(\sin (\phi)-\sin \left(\theta_{m}\right)\right)\right) y d_{y} \\
& =2 S e^{-j \psi_{0}} e^{-j \frac{2 \pi}{\lambda} F \frac{1-\cos \left(\theta_{m}\right)}{\cos \left(\theta_{m}\right)}} D_{z} \frac{1}{2 \pi / \lambda\left(\sin \left(\phi_{n}\right)-\sin \left(\theta_{m}\right)\right)} \\
& \times \int_{0}^{D_{y} / 2} \cos \left(2 \pi / \lambda\left(\sin (\phi)-\sin \left(\theta_{m}\right)\right)\right) y d_{\left(2 \pi / \lambda\left(\sin (\phi)-\sin \left(\theta_{m}\right)\right)\right) y} \\
& =S e^{-j \psi_{0}} e^{-j \frac{2 \pi}{\lambda} F \frac{1-\cos \left(\theta_{m}\right)}{\cos \left(\theta_{m}\right)}} D_{z} D_{y} \operatorname{sinc}\left(D_{y} / \lambda\left(\sin \left(\theta_{m}\right)-\sin (\phi)\right)\right) .
\end{aligned}
$$

Without loss of generality, we have assumed that $\psi_{0}=$ $2 n \pi$ for some integer $n$. Therefore, the phase term can be neglected and accordingly, we obtain

$Y\left(\theta_{m}\right)=S e^{-j \frac{2 \pi}{\lambda} F \frac{1-\cos \left(\theta_{m}\right)}{\cos \left(\theta_{m}\right)}} D_{z} D_{y} \operatorname{sinc}\left(D_{y} / \lambda\left(\sin \left(\theta_{m}\right)-\sin (\phi)\right)\right)$.

From (12), the array response of LNA can be obtained as

$$
a(\phi)_{m}=e^{-j \frac{2 \pi}{\lambda} F \frac{1-\cos \left(\theta_{m}\right)}{\cos \left(\theta_{m}\right)}} \operatorname{sinc}\left(D_{y} / \lambda\left(\sin \left(\theta_{m}\right)-\sin (\phi)\right)\right),
$$

where $\cos \left(\theta_{m}\right)=\frac{F}{\sqrt{F^{2}+(m d)^{2}}}$ and $\sin \left(\theta_{m}\right)=\frac{m d}{\sqrt{F^{2}+(m d)^{2}}}$. We assume that the steering vector of LNA is $\mathbf{a}(\phi)$ with its element $a(\phi)_{m}$, where $m \in[-(N-1) / 2, \ldots,(N-1) / 2]$. 
To simplify the presentation, the signal received through LNA can be denoted as

$$
\mathbf{y}(t)=p \mathbf{a}(\phi) e^{j b t}+\mathbf{n}(t),
$$

where $\mathbf{y} \in \mathbb{C}^{N \times 1}$ is the $N \times 1$ received signal vector, $p$ is the transmit signal amplitude, $b$ is the phase of the signal arrived at the lens, and $\mathbf{n}$ is the additive white Gaussian noise (AWGN) with covariance of $\sigma^{2} \mathbf{I}$, where $\mathbf{I}$ denotes the identity matrix.

The employment of large antenna arrays brings a number of new challenges. One of the most important challenges is that it increased signal processing complexity and hardware costs. In order to deal with such problem, antenna selection approach has received much attention $[12,13]$. Through antenna selection, the proposed algorithm will choose the best receiving antenna subset $G$ from the total receiving antennas $N$, where $G<<N$. This may reduce the number of required RF chains and the hardware costs. The best array element of LNA can be selected based on different criterias [12]. Since the lens has the ability of focusing the signal power on a subset of antennas as a function of DoAs, the maximal power approach can be tailored to choose an antennas which have the maximum power and its two nearby antennas. This can be expressed as:

$$
\breve{\mathbf{y}}=\breve{p} \breve{\mathbf{a}}(\phi) e^{j \breve{b} t}+\breve{\mathbf{n}}(t) \text {. }
$$

Subsequently, we may use the selected antennas to obtain the angles of the signals. Let the vector representation of the received signal for several selected antennas denote as

$$
\mathbf{x}(t)=\breve{\mathbf{y}} .
$$

In practice, the covariance matrix can be computed via the following sample averaging:

$$
\hat{\mathbf{R}}=\frac{1}{L} \sum_{t=1}^{L} \mathbf{x}(t) \mathbf{x}^{H}(t),
$$

where $L$ is the number of snapshots.

\section{Proposed algorithm}

In Section 2, we have derived an expression for the signal received out from the LNA structure. For the convergence of the $5 \mathrm{G}$ industries, it is very important to accurately estimating the DoAs of the signals and accordingly the directions of their sources. With this motivation, this paper focuses on evaluating the ML estimation for the DoA estimation using LNA. For the sake of simplicity, we define a parameter vector $\boldsymbol{\theta}=[p, b, \phi]$, where $p, b, \phi$ are the amplitude, phase, and DoAs of the received signal, respectively. By setting $\mathbf{v}=p \mathbf{a}(\phi) e^{b t}$, the probability density function (PDF) given the parameters vector $\boldsymbol{\theta}$ is represented as

$$
f_{x}(x \mid \boldsymbol{\theta})=c e^{(\mathbf{x}-\mathbf{v})^{H} \mathbf{R}^{-1}(\mathbf{x}-\mathbf{v})} .
$$

where $\mathbf{R}^{-1}=\sigma^{2} \mathbf{I}$ and $c$ is a normalization constant. After dropping the constant term, the log-likelihood of (18) becomes

$$
\begin{aligned}
g(p, \phi, b) & \triangleq \log \left(f_{x}(x \mid \boldsymbol{\theta})\right) \\
& =\frac{p}{\sigma^{2}}\left[p e^{-j b} \mathbf{a}(\phi)^{H} \mathbf{x}+p e^{j b} \mathbf{a}(\phi) \mathbf{x}^{H}-p \mathbf{a}^{H}(\phi) \mathbf{a}(\phi)\right] .
\end{aligned}
$$

To maximize the likelihood function, we assume that $b$ and $\phi$ are constants. The derivative of $p$ can be obtained as

$$
\frac{\partial g(p, \phi, b)}{\partial p}=\frac{1}{\sigma_{n}^{2}}\left[e^{-j b} \mathbf{a}^{H}(\phi) \mathbf{x}+e^{j b} \mathbf{a}(\phi) \mathbf{x}^{H}-\frac{2 p}{\sigma_{n}^{2}} \mathbf{a}(\phi)^{H} \mathbf{a}(\phi)\right],
$$

When the derivative equals to 0 , we can get

$$
p=\frac{e^{-j b} \mathbf{a}^{H}(\phi) \mathbf{x}+e^{j b} \mathbf{a}(\phi) \mathbf{x}^{2}}{2 \mathbf{a}^{H}(\phi) \mathbf{a}(\phi)} .
$$

Substitute (21) into (19), we have

$$
g(\phi, b)=\frac{\left(e^{-j b} \mathbf{a}^{H}(\phi) \mathbf{x}+e^{j b} \mathbf{a}(\phi) \mathbf{x}^{2}\right)^{2}}{4 \sigma^{2} \mathbf{a}^{H}(\phi) \mathbf{a} \phi} .
$$

By considering that $\phi$ is a constant, it follows form (21) that

$$
\frac{\partial g(\phi, b)}{\partial b}=-j e^{-2 j b}\left(\mathbf{a}^{H}(\phi) \mathbf{x}\right)^{2}+j e^{2 j b}\left(\mathbf{x}^{H} \mathbf{a}(\phi)\right)^{2} .
$$

Let $\mathbf{a}^{H}(\phi) \mathbf{x}$ equals $M e^{j \varphi}$, we obtain

$$
\frac{\partial g(\phi, b)}{\partial b}=-M^{2} e^{-2 j b} e^{j \varphi}+M^{2} e^{2 j b} e^{-j \varphi} .
$$

Then, when (24) equals to 0 , we can obtain

$$
b=\varphi .
$$

Substituting from (25) into (22), we can obtain

$$
g(\phi)=\frac{M^{2}}{\sigma^{2} \mathbf{a}^{2}}=\frac{\operatorname{tr}\left(\mathbf{a}^{H} \mathbf{x x}^{H} \mathbf{a}\right)}{\sigma^{2} \mathbf{a}^{H} \mathbf{a}} .
$$

For multiple samples, the likelihood function equals the sum of the likelihood functions for each sample. So we can get

$$
\begin{aligned}
\hat{\phi} & =\max _{\phi} \sum_{i=1}^{L} \frac{\operatorname{tr}\left(\mathbf{a}^{H} \mathbf{x}(i) \mathbf{x}(i)^{H} \mathbf{a}\right)}{\sigma^{2} \mathbf{a}^{2}} \\
& =\max _{\phi} \frac{\operatorname{tr}\left(\mathbf{a}^{H} \sum_{i=1}^{L} \mathbf{x}(i) \mathbf{x}(i)^{H} \mathbf{a}\right)}{\sigma^{2} \mathbf{a}^{2}} \\
& =\frac{\mathbf{a}^{H} R \mathbf{a}}{\sigma^{2} \mathbf{a}^{H} \mathbf{a}} .
\end{aligned}
$$

Up to now, we have presented the ML approach to DoA estimation using LNA. The proposed algorithm can be summarized as in Algorithm 1. 


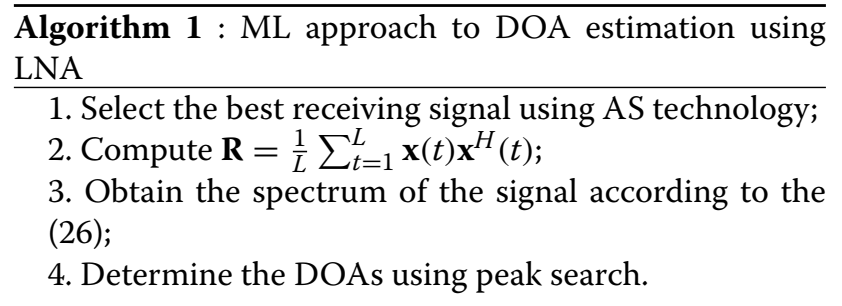

\section{Simulation results and discussion}

This section investigates the simulation result for the proposed ML approach for DoA estimation for the LNA structure. In all the experiments, we assume that the number of antenna elements $N=21$. The focal length $F=50 \lambda$ with $\lambda=10^{-3}$ and $D_{y}=10 \lambda$. The signal-noise ratio (SNR) is set to $0 \mathrm{~dB}$. Figure 2 compares the normalized power of received signals with LNA with that of the conventional schemes with uniform linear array (ULA) at different values for the angle of incident signals, namely $\alpha=-10^{\circ}$ and $\alpha=10^{\circ}$. It is proven that the power of received signals with ULA evenly distributed between all antenna elements, implying that the angle of the input signal has no effect on the power distribution. However, the power of received signal with LNA can be focused on a subset of array antenna and changes with the DoAs of incident signal. The reason behind this fact is that the receive array response vector of LNA is a sinc function of the DoA, which is an aperiodic function, while the response vector with ULA is a periodic function.

According to the characteristics of the power distribution of LNA, we propose using it to avoid the phase ambiguity problem. We assume that the DoAs of incident signals are in the range $\left[-10^{\circ}, 10^{\circ}\right]$. The obtained

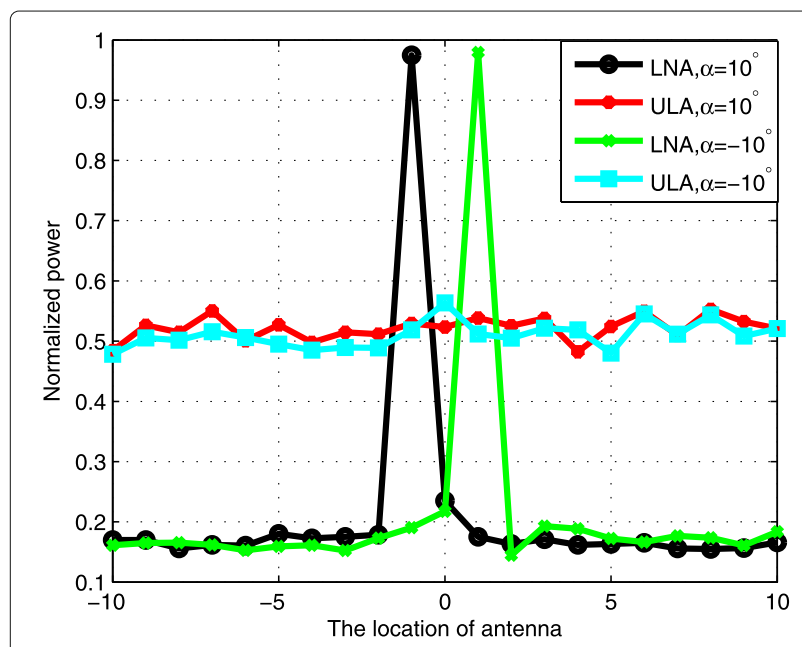

Fig. 2 Normalized power distribution comparison between LNA and ULA results are averaged over 100 simulation runs. The interelement spacing $d$ is assumed to be equal $\lambda$. Figure 3 shows the spatial spectrum of ML estimation with LNA and with ULA. The corresponding simulation result proves that the number of peaks with ULA and with LNA are 4 and 2, respectively. This is because the response vector with ULA is a periodic function. When $d$ is much larger than $\lambda / 2$, multiple angles can be achieved with the ML function, which causes the phase ambiguity. The response vector with LNA is sinc function. Regardless of the distance of antenna elements, there is only one angle to $\mathrm{ML}$ function. So it is easy to find the unique DoA and avoid phase ambiguity problem.

Although the array structure of LNA can solve the phase ambiguity problem, large antenna arrays require an equivalent large number of RF chains. In order to tackle this problem, this paper makes full use of the distribution characteristics of signal power and employs the maximal power approach to select the corresponding antenna and two antennas around it. In order to estimate the angle of the incident signal, we just need to deal with the data on these three antennas. In order to verify the feasibility of this selection criteria, we assume that $d$ equals to $\lambda / 2$ and $\alpha=10^{\circ}$. Spatial spectrum with ML estimation is illustrated in Fig. 3. The obtained results illustrate that the width of the main lobe using ULA with AS technique is greater than that achieved with other methods. This is because the power of received signals with ULA evenly distributed among all antenna elements, and the received signals using ULA with AS can lead to the lost of some parts of the signal received by the unselected antenna elements. The results also show that the width of the main lobe using LNA with AS is very close to the LNA without AS. Because LNA can focus the energy on a subset

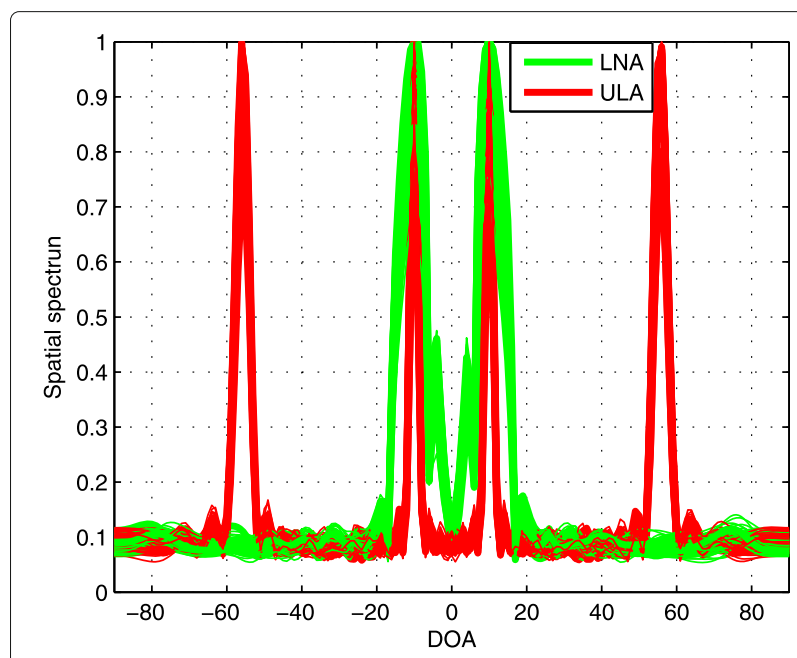

Fig. 3 Performance comparison of estimated DoA with and without EM lens 
of antennas and the information of the received signal using LNA with AS is almost concentrated in whole in the selected antenna subset. This indicates that the AS technique can be effectively applied to the LAN, but it is not useful in ULA. In addition, the estimation performance of the proposed method is evaluated by the root-meansquare error (RMSE) criteria, which is expressed as

$$
\mathrm{RMSE}=\frac{1}{P} \sum_{p=1}^{P} \sqrt{\frac{1}{K} \sum_{k=1}^{K}\left[\hat{\theta}_{k, p}-\theta_{k, p}\right]^{2}},
$$

where $P=100$ is the number of independent trials. The results in Figs. 4 and 5 show that the performance of using ULA with AS technique is worse than the other methods. However, the performance difference between the other three methods is not obvious. Therefore, it is necessary to discuss the situation of multiple incident signals.

In Fig. 6, we consider that there are two signals of $\boldsymbol{\theta}$ : $\left[-10^{\circ}, 10^{\circ}\right]$ and the SNR equals to $0 \mathrm{~dB}$. The results in this figure show that the ML approach using ULA with AS technique can not be used to estimate DoAs. This is because the resolution of DoA estimation is very low, when the number of selected antenna is three. Against this background, we would not consider the case of using ULA with AS techniques in that experiment as it is for sure will give worse performance that the case of LNA.

To further demonstrate the advantages of using LAN with AS techniques, we assume that there are four signals with DoAs of $\boldsymbol{\theta}:\left[-30^{\circ},-10^{\circ}, 10^{\circ}, 30^{\circ}\right]$. Figure 7 shows the performance of the proposed algorithm when SNR changes from $-5 \mathrm{~dB}$ to $5 \mathrm{~dB}$. It is also obvious from Fig. 7 that increasing the SNR leads to a monotonic decrease of the RMSE value. When the SNR $>1 \mathrm{~dB}$, the three methods in the comparison achieve an identical performance. When the SNR $<1 \mathrm{~dB}$, the conventional

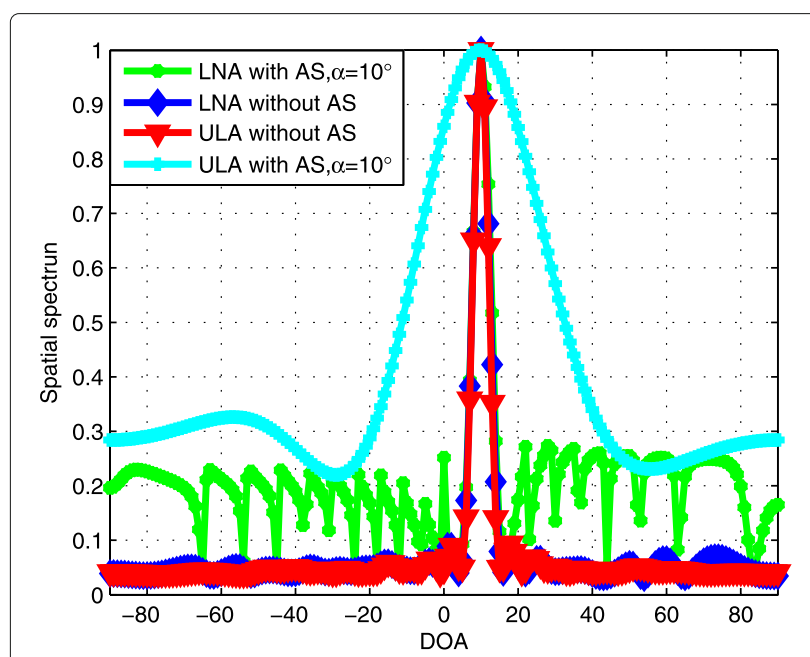

Fig. 4 Estimated DOA with and without EM lens

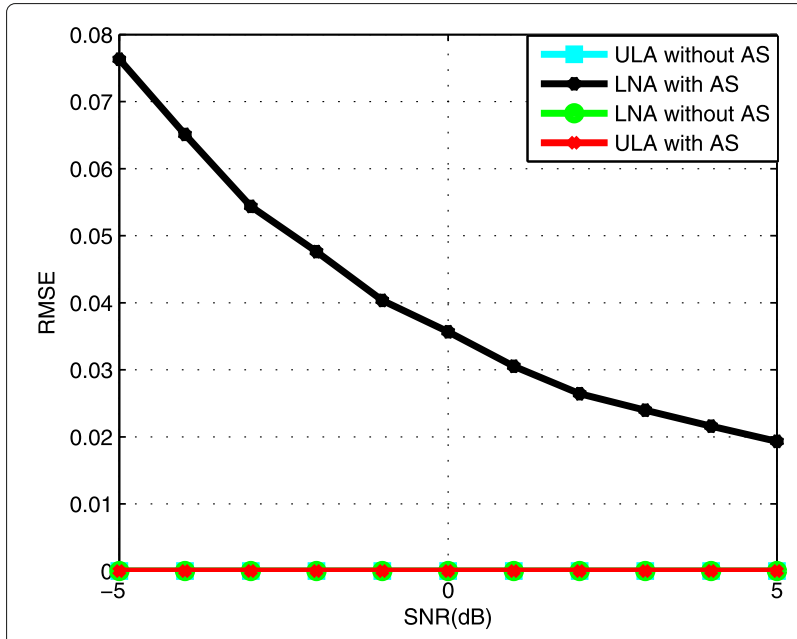

Fig. 5 RMSE of estimated DoA versus the SNR

scheme with ULA achieves better performance than the proposed method with LNA, and the proposed modification method with AS achieve the worst performance. This is because the systems with AS may lose copies of the signal received at other antenna elements. But the main advantage is that we just need to process the data out of only three antennas, which accordingly reduces signal processing complexity and hardware cost. In short, the proposed method is effective in estimating the DoAs of the signal, especially when SNR is very high.

\section{Conclusions}

We have proposed a ML approach for DoA estimation for the LNA structure. We have derived an expression for the received signal with LNA structure, and then a ML estimator for DoA estimation using LNA is also derived. One of the most important characteristic of LNA is the ability

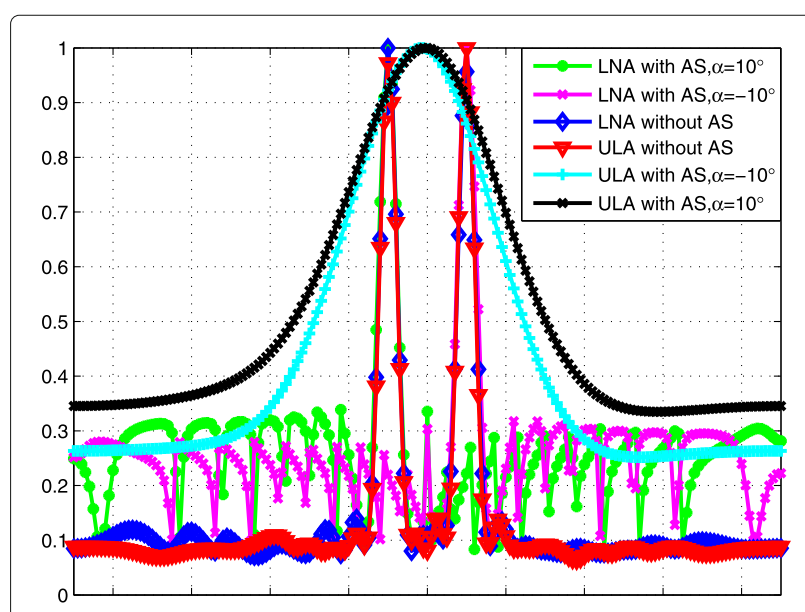

Fig. 6 Estimated DOA with and without EM lens 


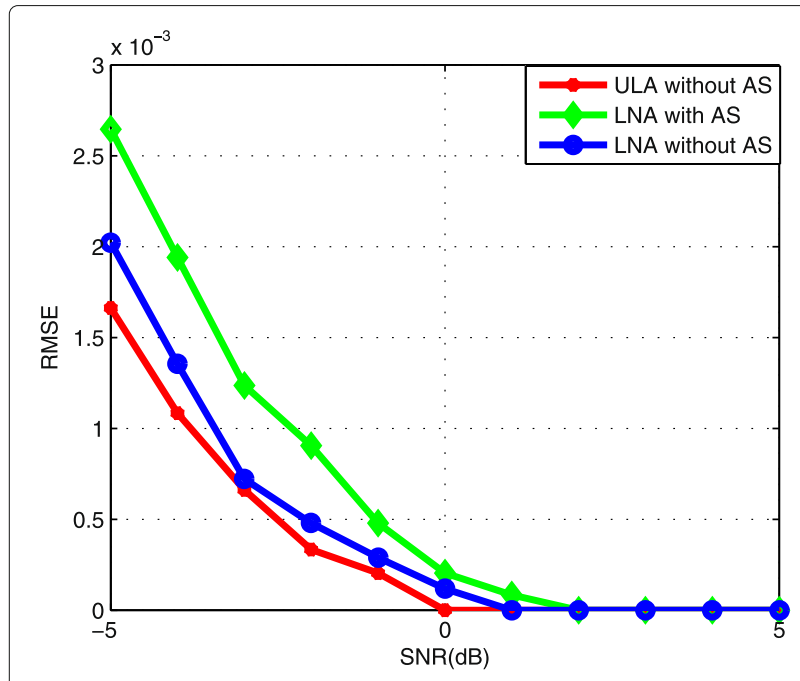

Fig. 7 RMSE of estimated DOAs versus the SNR

for focusing the energy. It is illustrated that the power distribution of receive signals is a sinc function of the incident signal direction. As a result, we for the first time, propose the use of LNA for estimating DoA and avoiding phase ambiguity. Moreover, we propose the employment of AS techniques to reduce the number of RF chains. Simulation results show that the proposed method can effectively estimate the DoA, avoiding phase ambiguity and decreasing the number of RF chains to a maximum of three RF chains. The results displayed in this paper can be used as a guideline for designing LNA for 5G systems' applications.

\section{Abbreviations}

A/D: Analog to digital; AS: Antenna selection; EM: Electromagnetic; D/A: Digital to analog; DOA: Direction-of-arrival; LNA: Lens antenna array; ML: Maximum likelihood; mmWave: Millimeter wave; MUSIC: Multiple signal classification; RF: Radio frequency

\section{Acknowledgements}

The authors would like to thank the anonymous reviewers for their insightful comments.

\section{Authors' contributions \\ All authors discussed the experiments. ZMJ performed the experiments and wrote the paper together with MR. Finally, PCZ, LH, and JHZ have made some useful comments on the paper. All authors have read and approved the final manuscript.}

\section{Funding}

This work is partially funded by Natural Science Foundation of China (NSFC), NO. 61601304, Foundation of Shenzhen funds witn No.

JCYJ20170302142545828, and 2016057.

\section{Availability of data and materials}

Not applicable.

\section{Competing interests}

The authors declare that they have no competing interests.

\section{Author details}

${ }^{1}$ Guangdong Laboratory of Artificial-Intelligence and Cyber- Economics (SZ), Shenzhen University, Shenzhen 518060, People's Republic of China. ${ }^{2}$ The
Department of Electronics and Electrical Communications Engineering, Faculty of Electronic Engineering, Menoufia University, Menouf 21974, Menoufia, Egypt.

Received: 20 September 2018 Accepted: 10 September 2019

Published online: 30 October 2019

\section{References}

1. J. G. Andrews, et al., What will $5 G$ be? IEEE J. Sel. Areas Commun. 32(6), 1065-1082 (2014)

2. Kwoczek A, Rech B, Hehn T, et al., 5G Automotive vision[J]. 5G Partnership Project (2015)

3. F. Boccardi, et al., Five disruptive technology directions for $5 \mathrm{G}$. IEEE Commun. Mag. 52(2), 74-80 (2014)

4. T. S. Rappaport, et al., Millimeter wave mobile communications for $5 \mathrm{G}$ cellular: It will work!. IEEE Access. 1(1), 335-349 (2013)

5. K.-T. Jo, et al., in 2009 IEEE Globecom Workshops. Multiple-antenna post low noise amplifier RF combining (IEEE, 2009). https://doi.org/10.1109/ glocomw.2009.5360684

6. Y. Zeng, R. Zhang, Z. N. Chen, Electromagnetic lens-focusing antenna enabled massive MIMO: Performance improvement and cost reduction. IEEE J. Sel. Areas Commun. 32(6), 1194-1206 (2014)

7. Y. Zeng, R. Zhang, Millimeter wave MIMO with lens antenna array: A new path division multiplexing paradigm. IEEE Trans. Commun. 64(4), 1557-1571 (2016)

8. X. Gao, et al., Massive MIMO in real propagation environments: Do all antennas contribute equally?. IEEE Trans. Commun. 63(11), 3917-3928 (2015)

9. X. Zhang, et al., Direction of departure (DOD) and direction of arrival (DOA) estimation in MIMO radar with reduced-dimension MUSIC. IEEE Commun. Lett. 14(12), 1161-1163 (2010)

10. P. Stoica, K. C. Sharman, Maximum likelihood methods for direction-of-arrival estimation. IEEE Trans. Acoust. Speech Signal Process. 38(7), 1132-1143 (1990)

11. M. A. Al-Joumayly, N. Behdad, Wideband planar microwave lenses using sub-wavelength spatial phase shifters. IEEE Trans. Antennas Propag. 59(12), 4542-4552 (2011)

12. S. Sanayei, A. Nosratinia, Antenna selection in MIMO systems. IEEE Commun. Mag. 42(10), 68-73 (2004)

13. P. Zhang, S. Chen, L. Hanzo, Two-tier channel estimation aided near-capacity MIMO transceivers relying on norm-based joint transmit and receive antenna selection. IEEE Trans. Wirel. Commun. 14(1), 122-137 (2015)

\section{Publisher's Note}

Springer Nature remains neutral with regard to jurisdictional claims in published maps and institutional affiliations.

\section{Submit your manuscript to a SpringerOpen ${ }^{\odot}$ journal and benefit from:}

- Convenient online submission

- Rigorous peer review

- Open access: articles freely available online

- High visibility within the field

- Retaining the copyright to your article

Submit your next manuscript at $>$ springeropen.com 